\title{
Equation of state effects in core-collapse supernovae
}

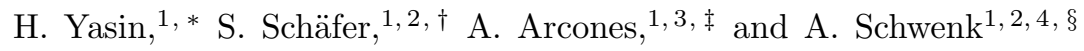 \\ ${ }^{1}$ Institut für Kernphysik, Technische Universität Darmstadt, 64289 Darmstadt, Germany \\ ${ }^{2}$ ExtreMe Matter Institute EMMI, GSI Helmholtzzentrum für Schwerionenforschung GmbH, 64291 Darmstadt, Germany \\ ${ }^{3}$ GSI Helmholtzzentrum für Schwerionenforschung GmbH, 64291 Darmstadt, Germany \\ ${ }^{4}$ Max-Planck-Institut für Kernphysik, Saupfercheckweg 1, 69117 Heidelberg, Germany
}

\begin{abstract}
We investigate the impact of different properties of the nuclear equation of state in core-collapse supernovae, with a focus on the proto-neutron-star contraction and its impact on the shock evolution. To this end, we introduce a range of equations of state that vary the nucleon effective mass, incompressibility, symmetry energy, and nuclear saturation point. This allows us to point to the different effects in changing these properties from the Lattimer and Swesty to the Shen et al. equations of state, the two most commonly used equations of state in simulations. In particular, we trace the contraction behavior to the effective mass, which determines the thermal nucleonic contributions to the equation of state. Larger effective masses lead to lower pressures at nuclear densities and a lower thermal index. This results in a more rapid contraction of the proto-neutron star and consequently higher neutrino energies, which aids the shock evolution to a faster explosion.
\end{abstract}

Core-collapse supernovae and neutron star mergers are cosmic laboratories for physics at the extremes. In the new multimessenger era, including also gravitational wave detection [1, we can uniquely combine observations and hydrodynamic simulations to learn more about these events. One critical microphysics input in simulations is the equation of state (EOS). In this Letter, we explore the macroscopic effects of the microphysics in the EOS in the context of supernova explosions.

Massive stars end their lives as core-collapse supernovae when their central iron cores collapse forming a proto-neutron star (PNS) and a shock wave that propagates through the infalling stellar layers. The final success of the shock to destroy the star depends on the neutrino energy deposited behind the shock. This is affected by convection, hydrodynamic instabilities, rotation, magnetic fields, and by the evolution of the PNS. Despite the many advances in simulating core-collapse supernovae including three-dimensional simulations (see, e.g., Refs. [28), the details about the explosion are still not clear.

The EOS is constrained by modern theoretical calculations at nuclear densities 920 , by nuclear experiments (see, e.g., Ref. 21 23]) as well as observations, in particular of two-solar-mass neutron stars 24, 25]. However, the properties of the EOS at densities above $(1-2) n_{0}$ (with saturation density $n_{0} \approx 0.16 \mathrm{fm}^{-3}$ ) remain quite uncertain, but these are relevant for simulations. There are two "classical" and commonly used EOSs in tabulated form, which cover the broad range of conditions reached in supernova simulations: the Lattimer and Swesty (LS) EOS [26, 27] and the H. Shen et al. (Shen) EOS [28. Recently, there have been major efforts to provide new EOS tables (see, e.g., Refs. 29 33).

A major impact of the EOS in supernova simulations is due to variations in the PNS contraction (see, e.g., Refs. [31, 34]). A faster contraction during the first few hundred milliseconds after bounce favors explosions due to higher neutrino energies and thus increased heating [34, 35]. This has been discussed when comparing different EOSs (including LS and Shen) 34 38. However, these studies are usually performed based on EOSs that differ in their underlying theoretical framework (based on Skyrme at high densities [26, 33 or relativistic energy-density functionals 28 32]) or within the same framework, varying all EOS parameters simultaneously 29, 31 33. This makes it difficult to link the behavior of the PNS and shock to a particular nuclear physics input. The only EOS work where solely one parameter was changed are those based on LS with different incompressibilities [26, which were applied, e.g., in Refs. [37, 38]. In this Letter, we individually vary different nuclear matter properties within the same EOS framework to clearly identify the impact of the effective mass, incompressibility, symmetry energy, and saturation point on the physics of core-collapse supernovae.

Equation of state and supernova simulations.- The LS EOS is based on a Skyrme energy-density functional, where the energy per nucleon of uniform matter as a function of baryon density $n$ and proton fraction $x=n_{p} / n$ at zero temperature is given by 26 .

$$
\begin{aligned}
\left.\frac{E}{A}\right|_{T=0}= & \frac{3 \hbar^{2}}{10 m^{*}}\left(3 \pi^{2} n\right)^{2 / 3}\left[(1-x)^{5 / 3}+x^{5 / 3}\right] \\
& +[a+4 b x(1-x)] n+c n^{\delta}-x \Delta .
\end{aligned}
$$

Here, $a, b, c$, and $\delta$ are the Skyrme parameters, and $\Delta$ is the neutron-proton mass difference. The nucleon effective mass $m^{*}$ is given by $\hbar^{2} /\left(2 m^{*}\right)=\hbar^{2} /(2 m)+\alpha n$, with $m=m_{n}=m_{p}=939.5654 \mathrm{MeV}$ in LS, and $\alpha$ is fit to the effective mass at saturation density.

In Table I, we list the EOS parameters for the LS EOS with incompressibility $K=220 \mathrm{MeV}$ (LS220) and the Shen EOS. We choose LS220 from the LS family, as this EOS supports a two-solar-mass neutron star and the incompressibility lies within the expected range from nuclear physics (see Table II). Moreover, Table II includes 


\begin{tabular}{l|cccc|cc}
\hline \hline & $m^{*} / m$ & $K$ & $E_{\text {sym }}$ & $L$ & $n_{0}$ & $B$ \\
\hline LS220 & 1.0 & 220 & 29.6 & 73.7 & 0.155 & 16.0 \\
Shen & 0.634 & 281 & $36.9^{\text {a }}$ & 110.8 & 0.145 & 16.3 \\
Theo. & $0.9(2)$ & $215(40)$ & $32(4)$ & $51(19)$ & $0.164(7)$ & $15.86(57)$ \\
\hline
\end{tabular}

a The symmetry energy in Shen is obtained via the second derivative of the energy per particle and not from the difference of neutron and symmetric matter as in LS.

TABLE I. Parameters for the LS220 and Shen EOS compared to theoretical ranges ("Theo.") from chiral EFT calculations for the effective mass $m^{*}$ at saturation density [16, 39, 40], incompressibility $K$ [17, 41, symmetry energy $E_{\mathrm{sym}}$ [11, 17, and $L$ parameter [11, 20] as well as the empirical ranges for the saturation density $n_{0}$ and energy $B$ given by the compilation in Ref. 20. All dimensionful quantities are in $\mathrm{MeV}$ except $n_{0}$ is in $\mathrm{fm}^{-3}$.

theoretical ranges from chiral EFT calculations and from the extraction of the empirical saturation point. As the effective mass is expected to be reduced at saturation density, we explore the impact of the effective mass by changing this from $m^{*}=m(\mathrm{LS} 220)$ to $m^{*} / m=0.8$ to $m^{*} / m=0.634$ (the Shen value). For the latter scenarios, we refit the Skyrme parameters $a, b, c$, and $\delta$ for given $m^{*}$ to reproduce the same saturation density $n_{0}$ and energy $B$, the incompressibility $K$, and symmetry energy $E_{\mathrm{sym}}$. This defines EOSs that are labeled as $m_{0.8}^{*}$ and $m_{\mathrm{S}}^{*}$, respectively. On top of $m_{\mathrm{S}}^{*}$, we vary the incompressibility $\left(m^{*}, K\right)_{\mathrm{S}}$, symmetry energy $\left(m^{*}, E_{\mathrm{sym}}\right)_{\mathrm{S}}$, and both $\left(m^{*}, K, E_{\mathrm{sym}}\right)_{\mathrm{S}}$ to the values of the Shen EOS. The EOS labeled SkShen additionally uses Shen values for saturation density and energy. In each case, the Skyrme parameters are refit so that the EOS parameters are varied one at a time. Finally, we note that the $L$ parameter, which determines the pressure of neutron matter, is not an independent parameter in the LS Skyrme functional (because there is only an isoscalar density-dependent $c$ term), but is determined by the other nuclear matter properties, such that the $L$ parameter varies for all constructed EOSs between the values of LS220 and Shen.

The EOS tables are created using the open-source code SROEOS from Ref. [33, 42, As a check, we also implemented an effective mass in the original code from LS [26, 43]. Both codes agree very well, except for small differences within the phase transition region (also discussed in Ref. [33]), but we have checked that these do not affect the findings of this Letter. As in Ref. [33, we refer to the LS220 generated EOS with the SROEOS code as LS220 ${ }^{\dagger}$. The Shen EOS table is taken from Ref. [44, 45].

The constructed EOSs offer novel insights into the impact of individual nuclear physics input on core-collapse supernovae. We perform spherically symmetric simulations with the FLASH code [46] for a $15 M_{\odot}$ progenitor [47. Moreover, we use the two-moment, energydependent, multispecies, neutrino radiation transport scheme M1 with an analytic closure [48 50, apply- ing the standard neutrino rates of Ref. [51, which include neutrino scattering on nucleons, alphas, and heavy nuclei, neutrino absorption on nucleons, neutrinoelectron inelastic scattering, electron-positron annihilation to neutrino-antineutrino pairs, as well as nucleonnucleon bremsstrahlung. The default NuLib rates are used (see Ref. [51] for further documentation). For every EOS, the neutrino opacity tables are created using Refs. [49, 51. Because spherically symmetric simulations do not explode for the chosen progenitor, we artificially increase the energy deposition by neutrinos in the gain layer by means of a heating factor. We emphasize that in multi-dimensional simulations the PNS is spherical even if there is convection inside (see, e.g., Ref. [52]). The use of spherically symmetric simulations to study the PNS behavior and its sensitivities to EOS parameters is therefore useful. However, the impact of convection needs to be also investigated as it may be relevant during the cooling phase [53]. The heating factor was chosen to produce an explosion for the LS220 ${ }^{\dagger}$ EOS.

Proto-neutron star and shock behavior.- Figure 1 shows the evolution of the PNS radius (defined as the position where the density is $10^{11} \mathrm{~g} \mathrm{~cm}^{-3}$ ) and shock radius post bounce (at $t=0 \mathrm{~s}$ ) based on the constructed EOSs that change the microphysics systematically from LS220 to Shen. The upper panel of Fig. 1 shows the critical impact of the effective mass on the PNS behavior, where $m^{*}$ mainly determines whether the contraction is faster (LS220 $0^{\dagger}$ with $m^{*}=m$ ), intermediate $\left(m_{0.8}^{*}\right)$, or slower (all other EOSs with $m_{\mathrm{S}}^{*}$ ). As discussed in more detail later (see Fig. 3), when the effective mass is reduced, the pressure increases $\left(P \sim 1 / m^{*}\right)$, and the observed behavior can be clearly traced to the thermal effects that depend on the effective mass and its density dependence. As shown by the shock evolution in the lower panel of Fig. 1. this has a direct impact on the explosion. When the effective mass is larger (LS220 ${ }^{\dagger}$ ) and the PNS contraction faster, the neutrino energies are higher. This leads to an earlier explosion and larger shock radii right after the explosion sets in 1

The impact of the incompressibility can be analyzed by comparing the EOS with $m_{\mathrm{S}}^{*}$ and $\left(m^{*}, K\right)_{\mathrm{S}}$ in Fig. 1 (see also Refs. 37, 38). The larger Shen incompressibility implies a higher pressure, which leads to a slightly larger PNS radius. However, this impact is much smaller compared to the changes due the effective mass. The symmetry energy has also a minor impact on the PNS and shock evolution, as is evident by comparing the EOS

\footnotetext{
${ }^{1}$ Note that the shock follows the evolution of the PNS 35 and thus the shock radius is smaller when the PNS contraction is stronger. This is found in non-exploding models. However, if the neutrino energies become high enough, then the explosion and expansion of the shock are stronger than this trend to follow the PNS evolution.
} 


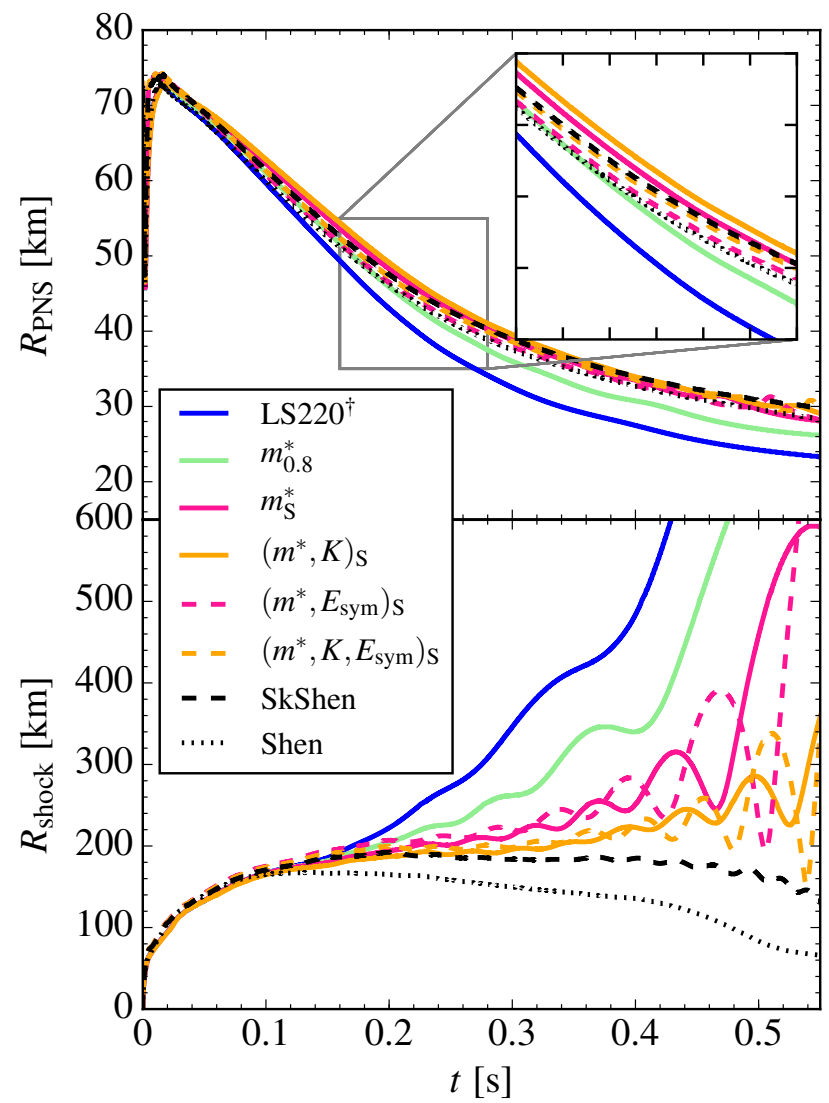

FIG. 1. Evolution of PNS radius (upper) and shock radius (lower panel) for supernova simulations of a $15 M_{\odot}$ progenitor based on EOSs with different microphysics properties ranging from LS220 ${ }^{\dagger}$ to Shen (as in the legend, for details see text). The shock presents an oscillatory behaviour for few models that are weakly exploding. This is due to the competition between accretion and explosion and it is overestimated in one-dimensional simulations.

with $m_{\mathrm{S}}^{*}$ to $\left(m^{*}, E_{\mathrm{sym}}\right)_{\mathrm{S}}$ and the EOS with $\left(m^{*}, K\right)_{\mathrm{S}}$ to $\left(m^{*}, K, E_{\mathrm{sym}}\right)_{\mathrm{S}}$. The symmetry energy variation of the PNS evolution is mainly due to the different conditions during collapse that result in a slightly larger electron fraction post-bounce for the models with higher symmetry energy (see also later, Fig. 2p. The SkShen EOS is as similar as possible to the Shen EOS in terms of the nuclear physics input; however the underlying framework is still different. The evolution of the shock is affected by several aspects besides the PNS contraction: different neutrino energies and luminosities because of variations in the interior PNS properties (see Fig. 2), bounce (initial time and position of the shock), as well as accretion evolution (see Refs. [31, 37] for a discussion). All this combined with the chosen heating factor contributes to the differences in shock evolution for the SkShen and Shen EOS. Nevertheless, qualitatively both SkShen and Shen evolutions are now more similar, especially for the shock behavior with an unsuccessful explosion.

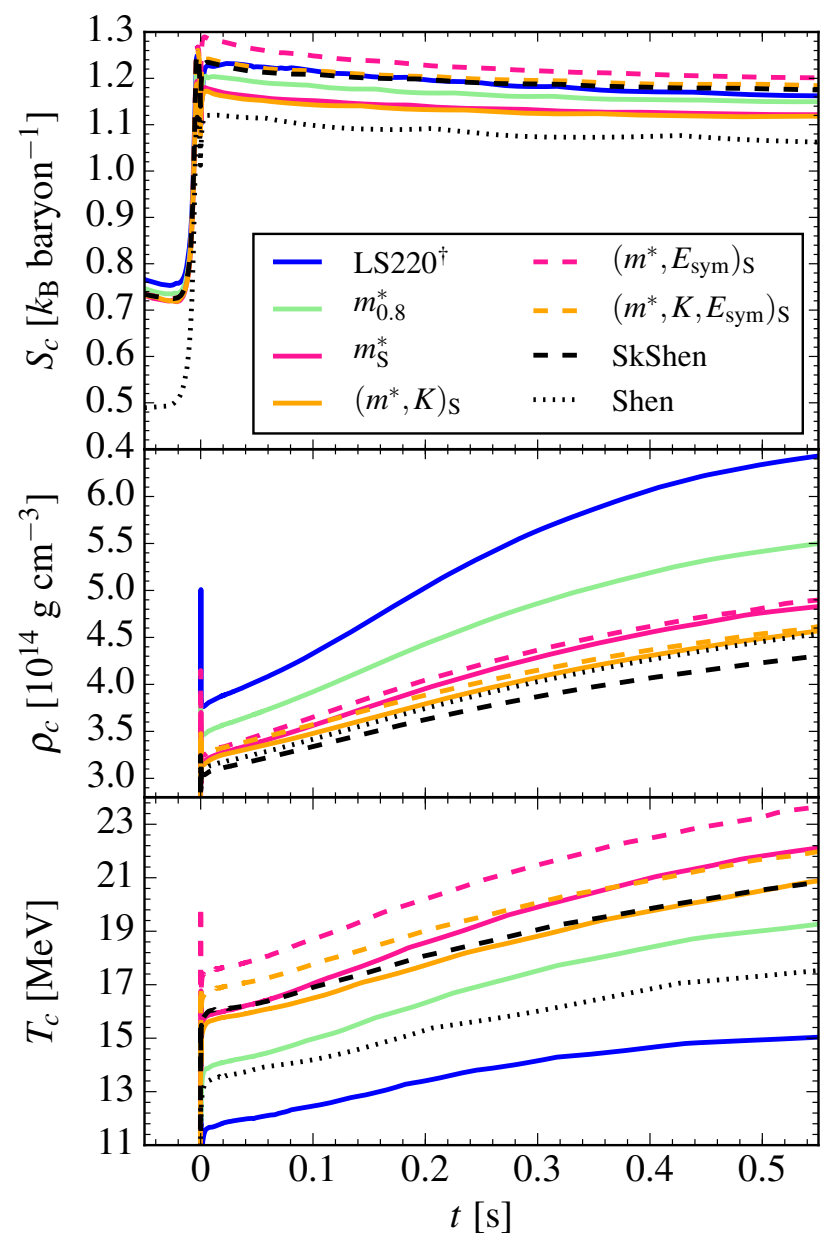

FIG. 2. Evolution of central values for the entropy (upper), density (middle), and temperature (lower panel) for the same simulations and EOSs as in Fig. 1 .

Impact of EOS on PNS interior.- To further study the impact of the different EOS parameters, we show the evolution of the central entropy, density, and temperature before and after bounce in Fig. 2 for the various EOSs considered. The central entropy (upper panel) only slightly depends on the effective mass. Note that the low central entropy obtained with the Shen EOS is due to the absence of kinetic entropy of nuclei [31]. The symmetry energy determines the electron fraction and entropy during collapse and after bounce [54, 55]. As shown in the top panel of Fig. 2, the EOSs with the lower symmetry energy have lower entropy and the post-bounce central electron fraction is $Y_{e, c} \approx 0.27$ compared to $Y_{e, c} \approx 0.30$ obtained for the higher Shen symmetry energy.

The central density (middle panel of Fig. 2 follows the effective mass hierarchy, because the pressure scales as $P_{c} \sim 1 / m^{*}$; this can further be seen in the top panel of Fig. 3. The PNS radii in Fig. 11 approximately follow the same hierarchy as the central density. Increasing the incompressibility and lowering the saturation density 
yields even higher central pressures, which in turn lowers the central density reached in the simulation.

The central temperature (lower panel in Fig. 2) is affected by changes in the effective mass as well as the symmetry energy. This can be understood considering that the entropy is approximately constant and independent of the EOS, and assuming a Fermi liquid theory scaling, $S_{c} \sim m^{*} T_{c} / \rho_{c}^{2 / 3}$ [56]. Reducing the effective mass thus increases the central temperature. Moreover, the larger value for the symmetry energy in the $\left(m^{*}, E_{\mathrm{sym}}\right)_{\mathrm{S}}$, $\left(m^{*}, K, E_{\mathrm{sym}}\right)_{\mathrm{S}}$, and SkShen EOS increases the central entropy and thus the central temperature. Similarly, the effect of the incompressibility can be understood through its impact on the central density. For the simulation based on the Shen EOS, the temperature is lower as expected from the entropy behavior discussed above.

Diagnosing thermal effects.- We have seen that the EOS impacts the interior of the PNS and thus the PNS contraction. Because $P_{c} \sim 1 / m^{*}$, we find larger central pressures for smaller $m^{*}$ as shown in the top panel of Fig. 3. The incompressibility determines the slope of the pressure, resulting in stiffer EOSs for the larger Shen incompressibility. In addition, the larger Shen symmetry energy yields even higher pressures, as this correlates with the $L$ parameter. The SkShen EOS results in the largest pressures of all our EOSs. This is due to the smaller saturation density, which leads to a larger pressure compared to an EOS starting from a higher $n_{0}$ (where $P=0$ ).

At the mean-field level in uniform matter, the thermal nucleonic contributions to the EOS are completely determined by the effective mass within the LS Skyrme functionals. In this approximation, the thermal index $\Gamma_{\text {th }}$ of a noninteracting gas of nonrelativistic fermions with density-dependent $m^{*}$ is given by (see, e.g., Ref. [57])

$$
\Gamma_{\mathrm{th}}=\frac{5}{3}-\frac{n}{m^{*}} \frac{\partial m^{*}}{\partial n} .
$$

We calculate $\Gamma_{\text {th }}$ from our simulations for all constructed EOSs by separating the pressure $P$ and energy density $\varepsilon$ into a cold and thermal (th) part following Ref. [58,

$$
\Gamma_{\mathrm{th}}=1+\frac{P_{\mathrm{th}}}{\varepsilon_{\mathrm{th}}}=1+\frac{P-P_{\text {cold }}}{\varepsilon-\varepsilon_{\text {cold }}}
$$

where we extract $P_{\text {cold }}$ and $\varepsilon_{\text {cold }}$ from the EOS table at the minimal temperature of $T=0.01 \mathrm{MeV}$. This is shown for the baryonic contributions only in the lower panel of Fig. 3. At high densities, we also compare this against $\Gamma_{\text {th }}$ of Eq. (2) shown as thick gray bands for the three different effective mass scenarios. The agreement is excellent, showing that a decreasing effective mass leads to a larger $\Gamma_{\text {th }}$ and thus a larger thermal contribution to the pressure. Note that SkShen has the same $m^{*}$ value at $n_{0}$, but a smaller saturation density, leading to a slightly larger $\Gamma_{\mathrm{th}}$ than the other $m_{\mathrm{S}}^{*}$ EOSs. The remaining differences to the Shen EOS are attributed to the underlying

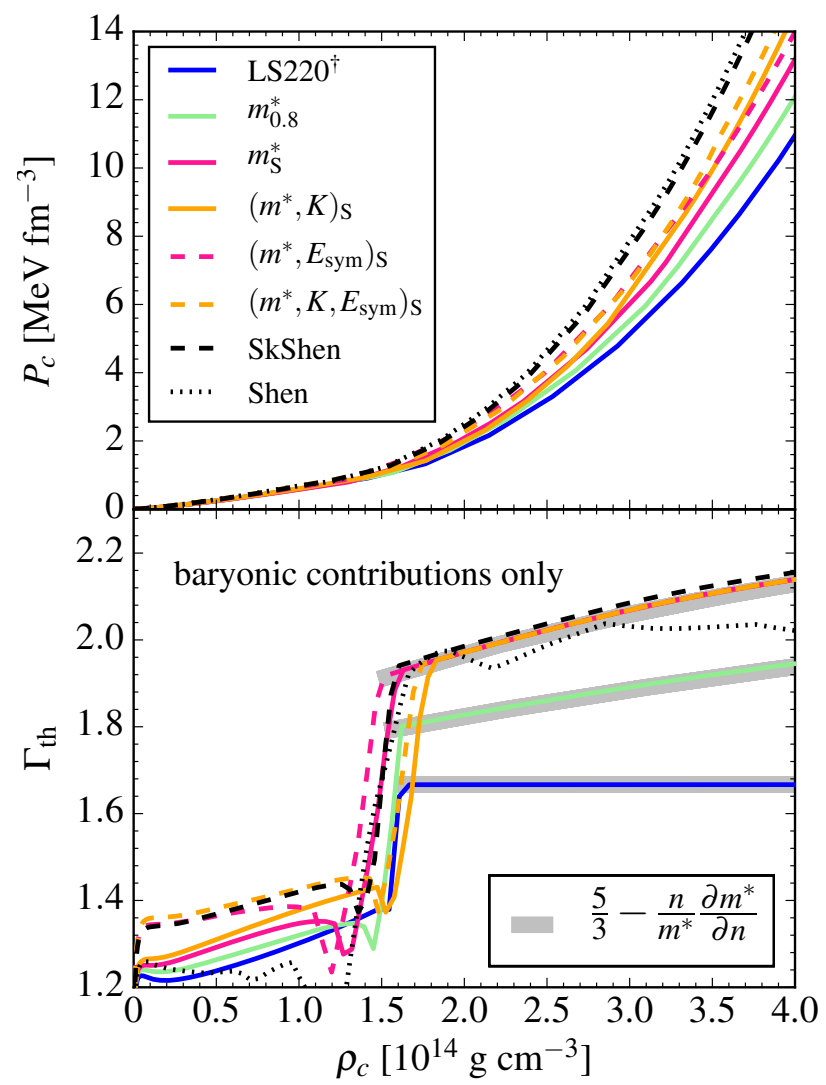

FIG. 3. Central pressure (upper) and thermal index $\Gamma_{\text {th }}$ (lower panel) as function of central density for the same simulations and EOSs as in Fig. 1] The results for $\Gamma_{\text {th }}$ are given for the baryonic contributions only, and are compared against $\Gamma_{\text {th }}$ of a noninteracting gas of nonrelativistic fermions with density-dependent $m^{*}$, Eq. 22, shown as thick gray bands at high densities for $m^{*}=m, m_{0.8}^{*}$, and $m_{\mathrm{S}}^{*}$.

relativistic mean-field formalism used. Below the phase transition, $\rho_{c} \lesssim 1.7 \mathrm{~g} \mathrm{~cm}^{-3}$, matter is no longer uniform and also clustering affects the thermal index.

Cold neutron stars.- Finally, we calculate the massradius $(M-R)$ relations for cold neutron stars to verify that the constructed EOSs give reasonable modifications to the $M-R$ relation. To this end, we solve the TolmanOppenheimer-Volkoff equations [59] for $T=0.1 \mathrm{MeV}$ and vanishing neutrino chemical potential. The results are shown in Fig. 4. All new EOSs are able to support a two-solar-mass neutron star 24, 25. Because the neutron star radius scales with the pressure of neutron matter at saturation density [22, 60, the radius and also the maximum mass in Fig. 4 increase with decreasing $m^{*}$ and larger incompressibility due to the larger pressures. Moreover, because the symmetry energy correlates with the $L$ parameter in the LS Skyrme model, we find that the radius increases significantly once the EOS used the large Shen symmetry energy. As the $L$ parameters for the EOSs constructed here are high compared to chiral EFT calculations (see Table I), the EOSs consid- 


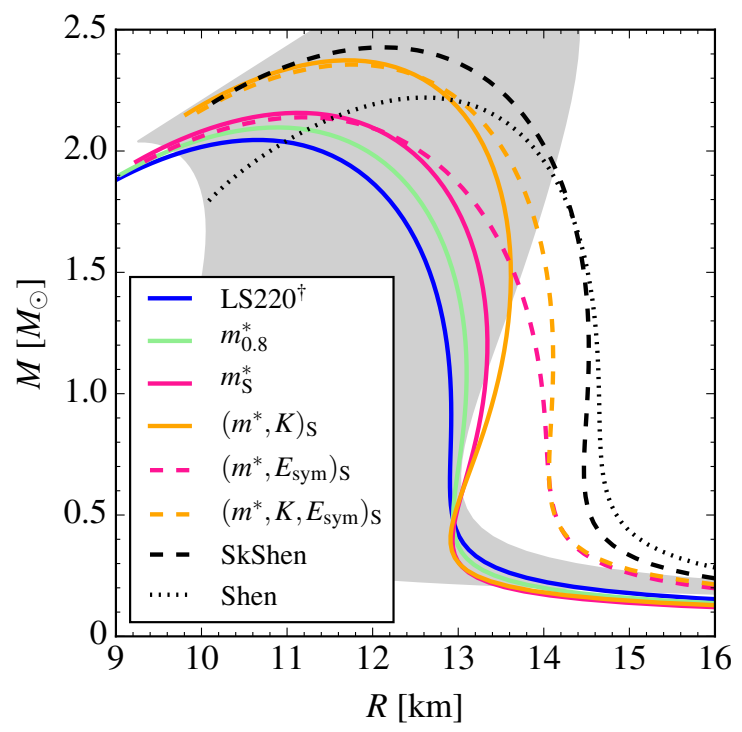

FIG. 4. Mass-radius relation for cold $(T=0.1 \mathrm{MeV})$ neutron stars in beta equilibrium for the various EOSs considered in this work. For comparison, we show the gray band from Ref. 11] based on chiral EFT calculations up to saturation density and a general extension to high densities.

ered lie towards larger radii compared to the gray band from Ref. [1] (see Fig. 4) based on chiral EFT calculations combined with a general extension to high densities. Moreover, it is reassuring that the SkShen EOS is similar to the relativistic energy-density functional based Shen EOS, once the same EOS parameters are used. This shows that indeed the physical properties are the important microphysics input and not the detailed scheme of the functional.

In summary, we have investigated core-collapse supernova simulations based on a range of EOSs by varying the nucleon effective mass, incompressibility, symmetry energy, and nuclear saturation point systematically from LS220 to Shen. All constructed EOS tables are available online [61]. In particular, we have shown that the effective mass has a decisive effect on the PNS contraction, with larger effective masses leading to a smaller thermal contribution to the pressure and thus a more rapid contraction. This aids the shock evolution to a faster explosion. By varying the EOS from LS220 to Shen, we were able to systematically step between these two commonly used EOSs and with SkShen show why the Shen EOS does not result in a successful explosion. While LS220 ${ }^{\dagger}$ was the EOS with the largest effective mass considered in this work, ab initio calculations of the EOS suggest that the effective mass can even increase to $m^{*}>m$ at higher densities due to contributions from correlations and three-nucleon forces 62. The effects also increased the radius of a cold $1.4 M_{\odot}$ neutron star from $12.8 \mathrm{~km}$ for LS220 $0^{\dagger}$ to $14.6 \mathrm{~km}$ for Shen, leading to a larger maximum mass as well. However, the EOS variation observed for the hot PNS radius clearly follows the behavior of the thermal effects diagnosed through the thermal index. Future work will include the construction of a range of EOSs based on existing and new chiral EFT constraints as well as further astrophysics explorations including also multi-dimensional simulations.

We thank A. Carbone, S. Greif, K. Hebeler, C. Mattes, E. O'Connor, and S. Couch for useful discussions. This work was supported by the Deutsche Forschungsgemeinschaft (DFG, German Research Foundation) - project-id 279384907 - SFB 1245 and the European Research Council Grant No. 677912 EUROPIUM.

* Email: hannah.yasin@physik.tu-darmstadt.de

$\dagger$ Email: sschaefer@theorie.ikp.physik.tu-darmstadt.de

¥ Email: almudena.arcones@physik.tu-darmstadt.de

$\S$ Email: schwenk@physik.tu-darmstadt.de

[1] B. P. Abbott et al. (The LIGO Scientific Collaboration and the Virgo Collaboration), Phys. Rev. Lett. 121, 161101 (2018)

[2] K. Kotake, K. Sumiyoshi, S. Yamada, T. Takiwaki, T. Kuroda, Y. Suwa, and H. Nagakura, Prog. Theor. Exp. Phys. 2012, 01A301 (2012)

[3] A. Burrows, Rev. Mod. Phys. 85, 245 (2013).

[4] E. J. Lentz, S. W. Bruenn, W. R. Hix, A. Mezzacappa, O. E. B. Messer, E. Endeve, J. M. Blondin, J. A. Harris, P. Marronetti, and K. N. Yakunin, Astrophys. J. Lett. 807, L31 (2015).

[5] B. Müller, Publ. Astron. Soc. Aust. 33, e048 (2016)

[6] H.-T. Janka, T. Melson, and A. Summa, Annu. Rev. Nucl. Part. Sci. 66, 341 (2016)

[7] R. Bollig, H.-T. Janka, A. Lohs, G. Martínez-Pinedo, C. J. Horowitz, and T. Melson, Phys. Rev. Lett. 119, $242702(2017)$

[8] E. O'Connor, R. Bollig, A. Burrows, S. Couch, T. Fischer, H.-T. Janka, K. Kotake, E. J. Lentz, M. Liebendörfer, O. E. B. Messer, A. Mezzacappa, T. Takiwaki, and D. Vartanyan, J. Phys. G 45, 104001 (2018)

[9] K. Hebeler and A. Schwenk, Phys. Rev. C 82, 014314 (2010).

[10] S. Gandolfi, J. Carlson, and S. Reddy, Phys. Rev. C 85, 032801(R) (2012)

[11] K. Hebeler, J. M. Lattimer, C. J. Pethick, and A. Schwenk, Astrophys. J. 773, 11 (2013).

[12] T. Krüger, I. Tews, K. Hebeler, and A. Schwenk, Phys. Rev. C 88, 025802 (2013).

[13] J. W. Holt, N. Kaiser, and W. Weise, Prog. Part. Nucl. Phys. 73, 35 (2013).

[14] A. Carbone, A. Polls, and A. Rios, Phys. Rev. C 88, 044302 (2013)

[15] G. Hagen, T. Papenbrock, A. Ekström, K. A. Wendt, G. Baardsen, S. Gandolfi, M. Hjorth-Jensen, and C. J. Horowitz, Phys. Rev. C 89, 014319 (2014)

[16] C. Wellenhofer, J. W. Holt, N. Kaiser, and W. Weise, Phys. Rev. C 89, 064009 (2014).

[17] C. Drischler, K. Hebeler, and A. Schwenk, Phys. Rev. C 93, 054314 (2016).

[18] J. E. Lynn, I. Tews, J. Carlson, S. Gandolfi, A. Gezerlis, 
K. E. Schmidt, and A. Schwenk, Phys. Rev. Lett. 116, 062501 (2016)

[19] I. Tews, J. Carlson, S. Gandolfi, and S. Reddy, Astrophys. J. 860, 149 (2018).

[20] C. Drischler, K. Hebeler, and A. Schwenk, Phys. Rev. Lett. 122, 042501 (2019)

[21] M. B. Tsang, J. R. Stone, F. Camera, P. Danielewicz, S. Gandolfi, K. Hebeler, C. J. Horowitz, J. Lee, W. G. Lynch, Z. Kohley, R. Lemmon, P. Möller, T. Murakami, S. Riordan, X. Roca-Maza, F. Sammarruca, A. W. Steiner, I. Vidaña, and S. J. Yennello, Phys. Rev. C 86, 015803 (2012).

[22] J. M. Lattimer and Y. Lim, Astrophys. J. 771, 51 (2013)

[23] J. Birkhan, M. Miorelli, S. Bacca, S. Bassauer, C. A. Bertulani, G. Hagen, H. Matsubara, P. von NeumannCosel, T. Papenbrock, N. Pietralla, V. Y. Ponomarev, A. Richter, A. Schwenk, and A. Tamii, Phys. Rev. Lett. 118, 252501 (2017)

[24] J. Antoniadis, P. C. C. Freire, N. Wex, T. M. Tauris, R. S. Lynch, M. H. van Kerkwijk, M. Kramer, C. Bassa, V. S. Dhillon, T. Driebe, J. W. T. Hessels, V. M. Kaspi, V. I. Kondratiev, N. Langer, T. R. Marsh, M. A. McLaughlin, T. T. Pennucci, S. M. Ransom, I. H. Stairs, J. van Leeuwen, J. P. W. Verbiest, and D. G. Whelan, Science 340, 1233232 (2013)

[25] E. Fonseca, T. T. Pennucci, J. A. Ellis, I. H. Stairs, D. J. Nice, S. M. Ransom, P. B. Demorest, Z. Arzoumanian, K. Crowter, T. Dolch, R. D. Ferdman, M. E. Gonzalez, G. Jones, M. L. Jones, M. T. Lam, L. Levin, M. A. McLaughlin, K. Stovall, J. K. Swiggum, and W. Zhu, Astrophys. J. 832, 167 (2016)

[26] J. M. Lattimer and F. D. Swesty, Nucl. Phys. A 535, 331 (1991)

[27] J. Lattimer, C. Pethick, D. Ravenhall, and D. Lamb, Nucl. Phys. A 432, 646 (1985)

[28] H. Shen, H. Toki, K. Oyamatsu, and K. Sumiyoshi, Nucl. Phys. A 637, 435 (1998)

[29] M. Hempel and J. Schaffner-Bielich, Nucl. Phys. A 837, $210(2010)$

[30] G. Shen, C. J. Horowitz, and S. Teige, Phys. Rev. C 83, 035802 (2011)

[31] M. Hempel, T. Fischer, J. Schaffner-Bielich, and M. Liebendörfer, Astrophys. J. 748, 70 (2012).

[32] A. W. Steiner, M. Hempel, and T. Fischer, Astrophys. J. 774, 17 (2013)

[33] A. S. Schneider, L. F. Roberts, and C. D. Ott, Phys. Rev. C 96, 065802 (2017).

[34] A. Marek, H. T. Janka, and E. Müller, Astronom. Astrophys. 496, 475 (2009)

[35] H.-T. Janka, Annu. Rev. Nucl. Part. Sc. 62, 407 (2012)

[36] K. Sumiyoshi, S. Yamada, H. Suzuki, H. Shen, S. Chiba, and H. Toki, Astrophys. J. 629, 922 (2005)

[37] S. M. Couch, Astrophys. J. 765, 29 (2013)
[38] Y. Suwa, T. Takiwaki, K. Kotake, T. Fischer, M. Liebendörfer, and K. Sato, Astrophys. J. 764, 99 (2013)

[39] K. Hebeler, T. Duguet, T. Lesinski, and A. Schwenk, Phys. Rev. C 80, 044321 (2009).

[40] C. Drischler, T. Krüger, K. Hebeler, and A. Schwenk, Phys. Rev. C 95, 024302 (2017).

[41] K. Hebeler, S. K. Bogner, R. J. Furnstahl, A. Nogga, and A. Schwenk, Phys. Rev. C 83, 031301(R) (2011)

[42] A. S. Schneider, L. F. Roberts, and C. D. Ott, https: //bitbucket.org/andschn/sroeos (2018).

[43] J. M. Lattimer and F. D. Swesty, http://www.astro. sunysb.edu/dswesty/lseos.html (2018).

[44] E. O'Connor and C. D. Ott, Class. Quant. Grav. 27, $114103(2010)$

[45] E. O'Connor and C. D. Ott, https://sntheory.org/ equationofstate (2018).

[46] B. Fryxell, K. Olson, P. Ricker, F. X. Timmes, M. Zingale, D. Q. Lamb, P. MacNeice, R. Rosner, J. W. Truran, and H. Tufo, Astrophys. J. Suppl. Ser. 131, 273 (2000).

[47] S. E. Woosley, A. Heger, and T. A. Weaver, Rev. Mod. Phys. 74, 1015 (2002)

[48] M. Shibata, K. Kiuchi, Y. Sekiguchi, and Y. Suwa, Prog. Theor. Phys. 125, 1255 (2011)

[49] E. O'Connor, Astrophys. J. Suppl. Ser. 219, 24 (2015)

[50] E. O'Connor and S. M. Couch, Astrophys. J. 854, 63 (2018)

[51] E. O'Connor, https://github.com/evanoconnor/nulib (2018).

[52] R. Buras, M. Rampp, H.-T. Janka, and K. Kifonidis, Astron. Astrophys. 447, 1049 (2006).

[53] L. F. Roberts, G. Shen, V. Cirigliano, J. A. Pons, S. Reddy, and S. E. Woosley, Phys. Rev. Lett. 108, 061103 (2012)

[54] H. Bethe, G. Brown, J. Applegate, and J. Lattimer, Nucl. Phys. A 324, 487 (1979).

[55] T. J. Mazurek, G. E. Brown, and J. M. Lattimer, Astrophys. J. 229, 713 (1979)

[56] G. Baym and C. J. Pethick, Landau Fermi-Liquid Theory (Wiley, New York, 1991).

[57] C. Constantinou, B. Muccioli, M. Prakash, and J. M. Lattimer, Phys. Rev. C 92, 025801 (2015).

[58] A. Bauswein, H.-T. Janka, and R. Oechslin, Phys. Rev. D 82, 084043 (2010)

[59] J. R. Oppenheimer and G. M. Volkoff, Phys. Rev. 55, 374 (1939).

[60] J. M. Lattimer and M. Prakash, Phys. Rep. 442, 109 (2007)

[61] H. Yasin, S. Schäfer, A. Arcones, and A. Schwenk, https://theorie.ikp.physik.tu-darmstadt.de/astro/ resources.php (2020).

[62] A. Carbone and A. Schwenk, Phys. Rev. C 100, 025805 (2019) 